\title{
Predicting broad-spectrum antiviral drugs against RNA viruses using transcriptional responses to exogenous RNA
}

Akpeli V. Nordor ${ }^{*}$, Geoffrey H. Siwo 2,3

\author{
Affiliations: \\ 1Institut Curie, Paris, France \\ 2Eck Institute for Global Health, Department of Biological Sciences, University of Notre Dame, \\ IN, 46556, USA \\ ${ }_{3}$ Center for Research Computing, University of Notre Dame, IN, 46556, USA
}

${ }^{*}$ Current affiliation: Epigene Labs, Paris, France

To whom correspondence should be addressed: gsiwo@nd.edu

\begin{abstract}
All RNA viruses deliver their genomes into target host cells through processes distinct from normal trafficking of cellular RNA transcripts. The delivery of viral RNA into most cells hence triggers innate antiviral defenses that recognize viral RNA as foreign. In turn, viruses have evolved mechanisms to subvert these defenses, allowing them to thrive in target cells. Therefore, drugs activating defense to exogenous RNA could serve as broadspectrum antiviral drugs. Here we show that transcriptional signatures associated with cellular responses to the delivery of a non-viral exogenous RNA sequence into human cells predicts small molecules with broad-spectrum antiviral activity. In particular,
\end{abstract}


transcriptional responses to the delivery of cas9 mRNA into human hematopoietic stem and progenitor cells (HSPCs) highly matches those triggered by small molecules with broad-spectrum antiviral activity such as emetine, homoharringtonine, pyrvinium pamoate and anisomycin, indicating that these drugs are potentially active against other RNA viruses. Furthermore, these drugs have been approved for other indications and could thereby be repurposed to novel viruses. We propose that the antiviral activity of these drugs to SARS-CoV-2 should therefore be determined as they have been shown as active against other coronaviruses including SARS-CoV and MERS-CoV. These drugs could also be explored as potential adjuvants to COVID-19 vaccines in development due to their potential effect on the innate antiviral defenses that could bolster adaptive immunity when delivered alongside vaccine antigens.

\section{Introduction}

Invasion by viruses presents one of the biggest challenges faced by both prokaryotic and eukaryotic cells. As a consequence, a wide range of mechanisms to counter viral infections have evolved across all life forms. In higher vertebrates including mammals, both innate and acquired immunity to viruses are employed as a defense against viral infections. While acquired immunity is driven by highly specialized cell types, for example B and T-cells, innate immunity against viral infections is possessed by nearly all cell types in mammals. Unlike acquired immunity which involves antigen-specific receptors generated through gene rearrangements, innate defense against a broad range of viruses relies on germline encoded pattern recognition receptors (PRRs). Antiviral defenses by PRRs recognize specific pathogen associated molecular patterns (PAMPs) that are 
crucial for viral replication and transmission, for example viral nucleic acid (1), and hence difficult for the target virus to change. However, many viruses circumvent this challenge by targeting PRPs. Thus, activating the intrinsic antiviral defenses in individual cells could provide a means for developing broad-spectrum antiviral drugs.

Here, we hypothesized that the delivery of an exogenous RNA sequence into human cells elicits antiviral defense characterized by a specific transcriptional response, and that small molecules that enhance this response have potential for broad-spectrum antiviral activity. In contrast to viruses which subvert innate cellular defenses to foreign RNA, nonviral exogenous RNA should evoke antiviral responses but fail to subvert these defenses. Thus, studying cellular responses to non-viral exogenous RNA could provide an unbiased view of exogenous RNA defenses. Using the Connectivity Map database of thousands of genetic and chemical perturbations of human cell lines (2), we show that several small molecules with broad-spectrum antiviral activity elicit transcriptional responses that are highly similar to those of human cells perturbed by the delivery of an exogenous, synthetic RNA sequence.

\section{Results}

\section{Relationship between transcriptional responses to exogenous RNA and viral infection}

As a proxy to cellular response to the delivery of exogenous RNA, we considered recently published transcriptional responses of a human cell type to delivery of non-viral RNA sequences encoding cas9 protein and CRISPR gRNA to hemoglobin B locus (HBB), 
hereafter referred to as 'cas9 mRNA' (3). Specifically, the delivery of cas9 mRNA into hematopoietic stem and progenitor cells (HSPCs) was found to induce a strong antiviral transcriptional response (3). Several genes involved in innate antiviral response such as interferon stimulatory genes (ISGs), interferon regulatory factors (IRF1, IRF7, IRF9) and the cytosolic foreign RNA sensor RIG-1 were upregulated by cas9 mRNA delivery (3). The activation of innate antiviral defenses upon delivery of exogenous RNA into cells has also been reported for in vitro transcribed CRISPR guide RNAs (4). As little as $1 \mathrm{~nm}$ of gRNA triggered a 30 to 50 fold increase in interferon-beta1 (IFNB1) in HEK293T cells and $50 \mathrm{~nm}$ gRNA led to a 1000 -fold induction of IFNB1 (4), an effect that is equal to that induced by RNA from Sendai virus or hepatitis C virus PAMP $(5,6)$.

To explore the potential regulatory mechanisms underlying transcriptional response to cas9 mRNA delivery and their association with key antiviral response regulators, we investigated the similarity of the observed transcriptional changes to those obtained under overexpression of thousands of genes across different cell lines in the Connectivity Map (CMap) database, a compendium of more than 1 million transcriptional profiles of human cell lines under thousands of chemical and genetic perturbations (2) (Fig. 1 and Methods). Interestingly, we found that the overexpression of IFNB1 across several cell lines in the CMap database has a transcriptional signature that is the most similar to the delivery of cas9 mRNA (Fig. 1), consistent with previous reports that CRISPR gRNAs induce IFNB1 $(4,7)$. In addition, several of the top ranked signatures are of genes involved in innate immunity to viruses such as B-cell lymphoma-2-like protein (BCL2L2), TNFRFS1A, KLF6 and TIRAP. For example, B-cell lymphoma-2-like protein (BCL2L2) inhibition by the 
anticancer compound ABT-263 accelerates apoptosis of influenza A virus (IAV) infected cells and lowers survival of infected mice (8); Kruppel-like factor 6 (KLF6) is a transcription factor that induces the production of the antiviral compound nitric oxide during infection by negative-sense, single stranded RNA viruses such as IAV (9) or respiratory syncytial virus (RSV) (10); BCL10 is required for antiviral response mediated by the retinoic acid inducible gene 1 (RIG-1) which senses foreign RNA in the cytosol $(11,12)$, while the tollinterleukin-1 domain adaptor receptor containing protein (TIRAP) is required for innate immune signaling to ligands such as viral nucleic acids in multiple subcellular compartments (13).

\section{Cell lines}

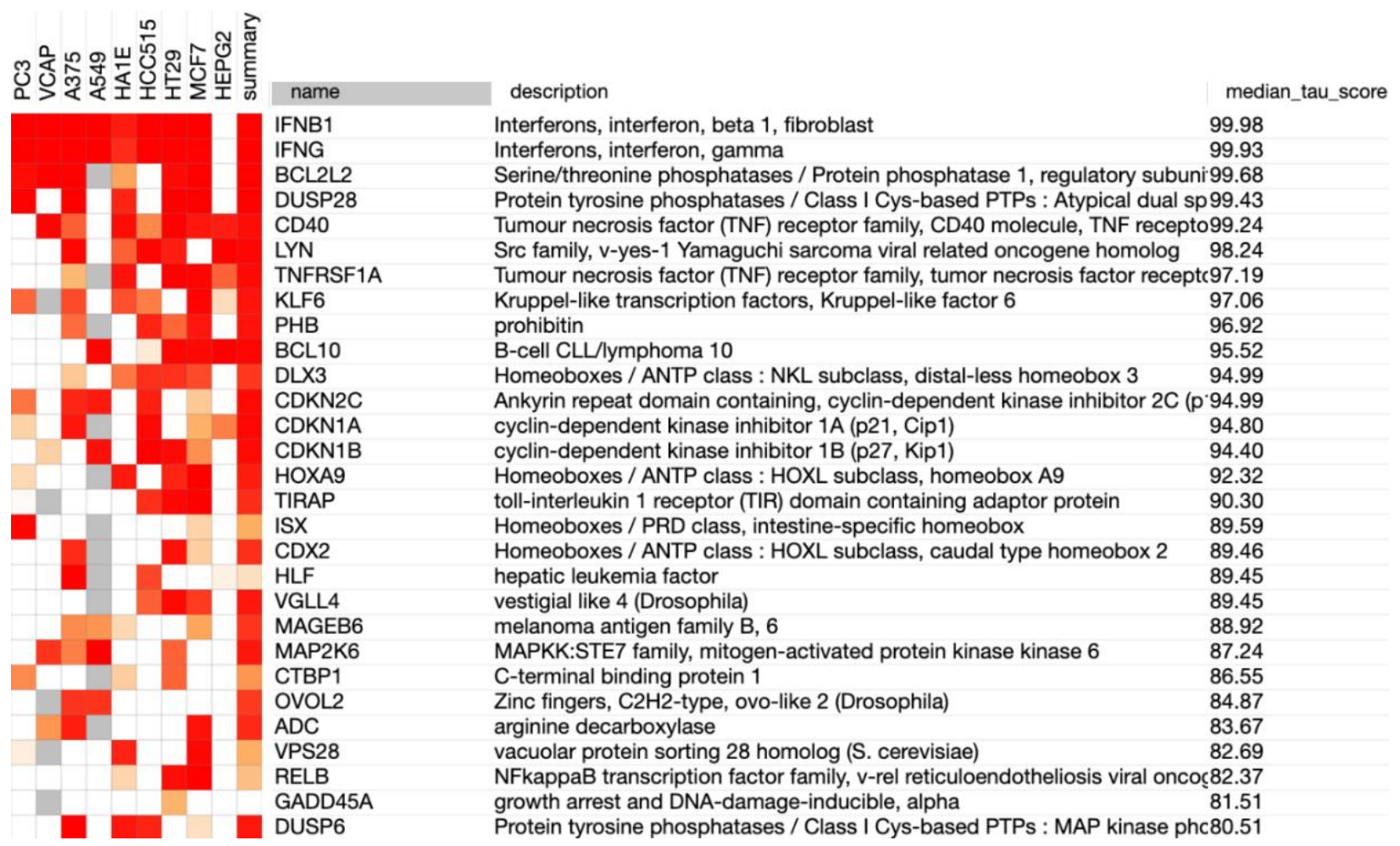

Fig 1: Genes whose over-expression in different cell lines matches transcriptional signatures of the delivery of cas9 mRNA into HSPCs. The median score measures the 
statistical significance of the similarity between the query signature (in this case cas9 mRNA signature) and the signatures in the database. The cas9 mRNA sequences consisted of a set of upregulated and downregulated genes following cas9 mRNA and CRISPR sgRNA delivery into HSPCs using previously published data (3). A high positive similarity implies that the transcriptional signatures are highly similar, i.e. upregulated genes in the query also tend to be upregulated in the matching CMap database experiment and downregulated genes in the query tend to be downregulated in the CMap experiment. The summary score shows the overall similarity of the query signature to the experiments across various human cell lines.

\section{Similarity between transcriptional signatures to exogenous RNA and small molecules}

Next, we investigated the similarity between transcriptional response to cas9 mRNA delivery and small molecules in the CMap database. We have previously shown that the transcriptional signatures of several small molecules in various cell lines are highly similar to those associated with the delivery of CRISPR/Cas9 components, including the delivery of cas9 mRNA sequence (14). Given that transcriptional response to cas9 mRNA is associated with antiviral transcriptional signatures, we reasoned that some of the small molecules with transcriptional signatures similar to that of cas9 mRNA could bear broadspectrum antiviral activity. Since the delivery of cas9 mRNA may also lead to transcriptional responses unrelated to foreign RNA sensing, we filtered out small molecules whose transcriptional signatures are highly similar to that of the delivery of cas9 protein pre-complexed with gRNA to form a ribonucleoprotein, RNP (Fig. 2). This 
process removed small molecules such as etoposide, teniposide and idarubicin which may have similar transcriptional effects to cas 9 mRNA due to downstream effects of cas 9 protein expression in inducing DNA cleavage. Notably, the top small molecules remaining after this filtering included several protein synthesis inhibitors namely puromycin, homoharringtonine, emetine (and its structural analog cephaeline), anisomycin and cycloheximide (Fig. 2). Except for puromycin, these protein synthesis inhibitors have been shown to have selective activity towards several viruses. At very low concentrations where they do not significantly affect host protein synthesis and cell viability, cycloheximide and anisomycin mimic interferon treatment by inhibiting the synthesis of specific proteins of encephalomyocarditis virus, murine leukemia virus and vesicular stomatitis virus $(15,16)$. Homoharringtonine is a broad-spectrum antiviral molecule that enhances the degradation of phosphorylated eukaryotic initiation factor 4E (elF4E) which is required for the synthesis of viral proteins (17) while emetine shows broad-spectrum antiviral activity to DNA and RNA viruses such as ebola virus, zika virus, bovine herpesvirus-1 and Newcastle disease virus $(18,19)$. Translation initiation and elongation are obligate steps in the replication cycle of many fast replicating RNA viruses, thus these small molecules could inhibit several RNA viruses. Interestingly, elF4E enhances the antiproliferative effects of IFN by promoting the translation of interferon stimulated genes (ISGs) demonstrating that translational control is an important part of the innate antiviral defense (20). In addition to the protein synthesis inhibitors, we found that thapsigargin an inhibitor of sarco/endoplasmic reticulum Ca2+ ATPase (SERCA) also has transcriptional signatures that highly match those of cas9 mRNA delivery. The inhibition of SERCA by thapsigargin results into endoplasmic reticulum (ER) stress leading to the 
activation of interferon regulatory factor 3 (IRF3), a transcription factor that regulates multiple IFN-inducing pathways triggered by DNA and RNA viruses (21). Thapsigargin has antiviral activity at low concentrations without cellular toxicity to several viruses including Newcastle disease virus, peste des petits ruminant virus, murine norovirus and flaviviruses (22-24). Narciclasine, another top small molecule (Fig. 2) has been shown to be active against the RNA flaviviruses (Japanese encephalitis, yellow fever and dengue viruses) as well as to the bunyaviruses Punta Toro and Rift Valley Fever virus (25).

\section{Implications for broad-spectrum antivirals to emerging viruses, SARS-CoV-2}

There is an urgent need to identify broad-spectrum antiviral drugs to emerging viral infections. In particular, the recent emergence of the SARS-CoV-2 virus and its rapid spread through all parts of the world demands fast development of antiviral drugs and vaccines to counter the ensuing COVID-19 pandemic $(26,27)$. To explore the potential for the predicted small molecules to inhibit SARS-CoV-2 in addition to their known broadspectrum antiviral effects, we searched published literature on their potential activity on other coronaviruses including SARS-CoV, MERS-CoV, HCoV-NL63, HCoV-OC43 and HCoV-229E, mined a public database of broad-spectrum antiviral agents (28) and considered whether these molecules have been approved for other diseases. In a recent high-throughput screen of a library of 2,000 approved and pharmacologically active compounds, emetine, pyrvinium pamoate and cycloheximide were found to have broadspectrum activity against the coronaviruses MERS-CoV, HCoV-OC43, HCoV-NL63 and MHV-A59 at low micromolar concentrations (29). Furthermore, in a separate screen of a library of 290 compounds with FDA approval or advanced clinical development, emetine, 
anisomycin, cycloheximide and homoharringtonine had high activity to both MERS-CoV and SARS-CoV (30). Emetine is approved in many countries for the treatment of amoebic dysentery, homoharringtonine has been used for 30 years in China for the treatment of several tumors and a semisynthetic derivative of the drug was approved by the US FDA in 2012 for the treatment of chronic myelogenous leukemia (CML) while pyrvinium pamoate is approved by the FDA for the treatment of helminth infections. Notably, out of the compounds screened for activity against MERS-CoV, anisomycin and emetine had the lowest $\mathrm{EC}_{50}$ while for SARS-CoV cycloheximide and emetine had the lowest $\mathrm{EC}_{50}$ in a previous study (30). Thus, the predicted molecules appear to have high activity to these RNA viruses (Fig.2).

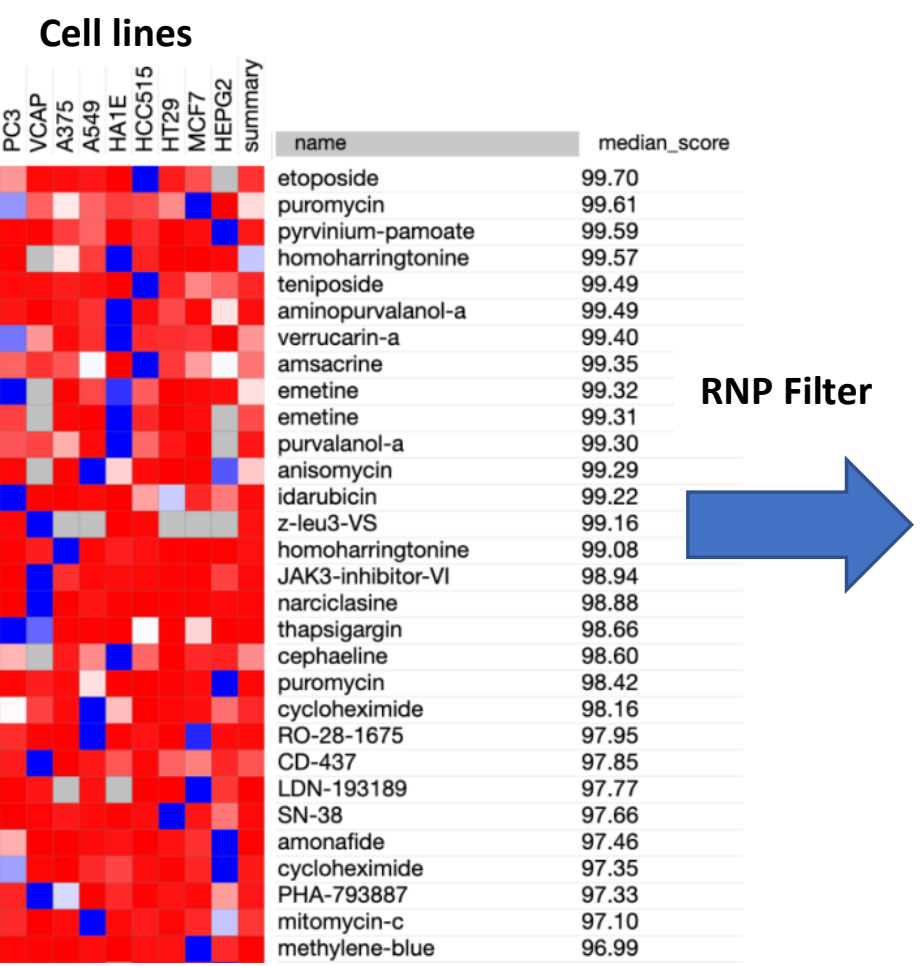

\begin{tabular}{lll}
\hline Drug & $\begin{array}{l}\text { SARS- } \\
\text { CoV EC50 }\end{array}$ & $\begin{array}{l}\text { MERS- } \\
\text { CoV EC } 50\end{array}$ \\
\hline $\begin{array}{l}\text { Pyrvinium- } \\
\text { pamoate }\end{array}$ & & 1.84 \\
$\begin{array}{l}\text { Homoharringt- } \\
\text { onine }\end{array}$ & 0.0718 \\
\hline $\begin{array}{l}\text { Emetine } \\
\text { Anisomycin }\end{array}$ & 0.191 & 0.003 \\
\hline Cycloheximide & 0.043 & 0.189 \\
\hline
\end{tabular}

Fig. 2: Small molecules whose transcriptional signatures in various cell lines match those of HSPC response to cas9 mRNA delivery. The list of molecules was filtered to exclude 
DNA repair inhibitors (etoposide, teniposide, idarubicin) and further refined to select molecules with evidence of activity in SARS-CoV and MERS-CoV. The EC50 for pyrvinium pamoate are based on published data from a high-throughput screen of a library containing 2000 compounds (29) while EC50 for emetine, anisomycin, homoharringtonine and cycloheximide are based on a screen of 290 approved compounds (30).

A number of broad-spectrum antiviral agents are currently being tested for the ongoing COVID-19 pandemic including remdesivir, chloroquine and ritonavir. Remdesivir (GS5734), a nucleoside analog prodrug developed by Gilead Sciences is a promising antiviral agent that has been shown to inhibit other coronaviruses including MERS-CoV (31), SARS-CoV-2 in vitro (32) and in the first patient of COVID-19 in the United States (33). Chloroquine, a onetime successful anti-malarial drug, has also shown activity against SARS-CoV-2 and other viruses $(32,34)$. We compared the broad-spectrum antiviral range of these drugs to those of the small molecules identified in this study (Fig. 3). Unlike remdesivir which is undergoing a clinical trial for SARS-CoV-2, the drugs we identified in this study have a number of advantages: i) they target host antiviral defenses instead of viral proteins, and may therefore avoid evolution of drug resistance which is rampant in RNA viruses, ii) they are likely to have activity across viruses that have little genetic similarity and infect different cell types, and iii) compared to other drugs that are only active in cells they diffuse in, small molecules that activate antiviral defenses could result into secretion of antiviral effector proteins, cytokines and chemokines that diffuse to other cells and attract/ activate specialized immune cells. 

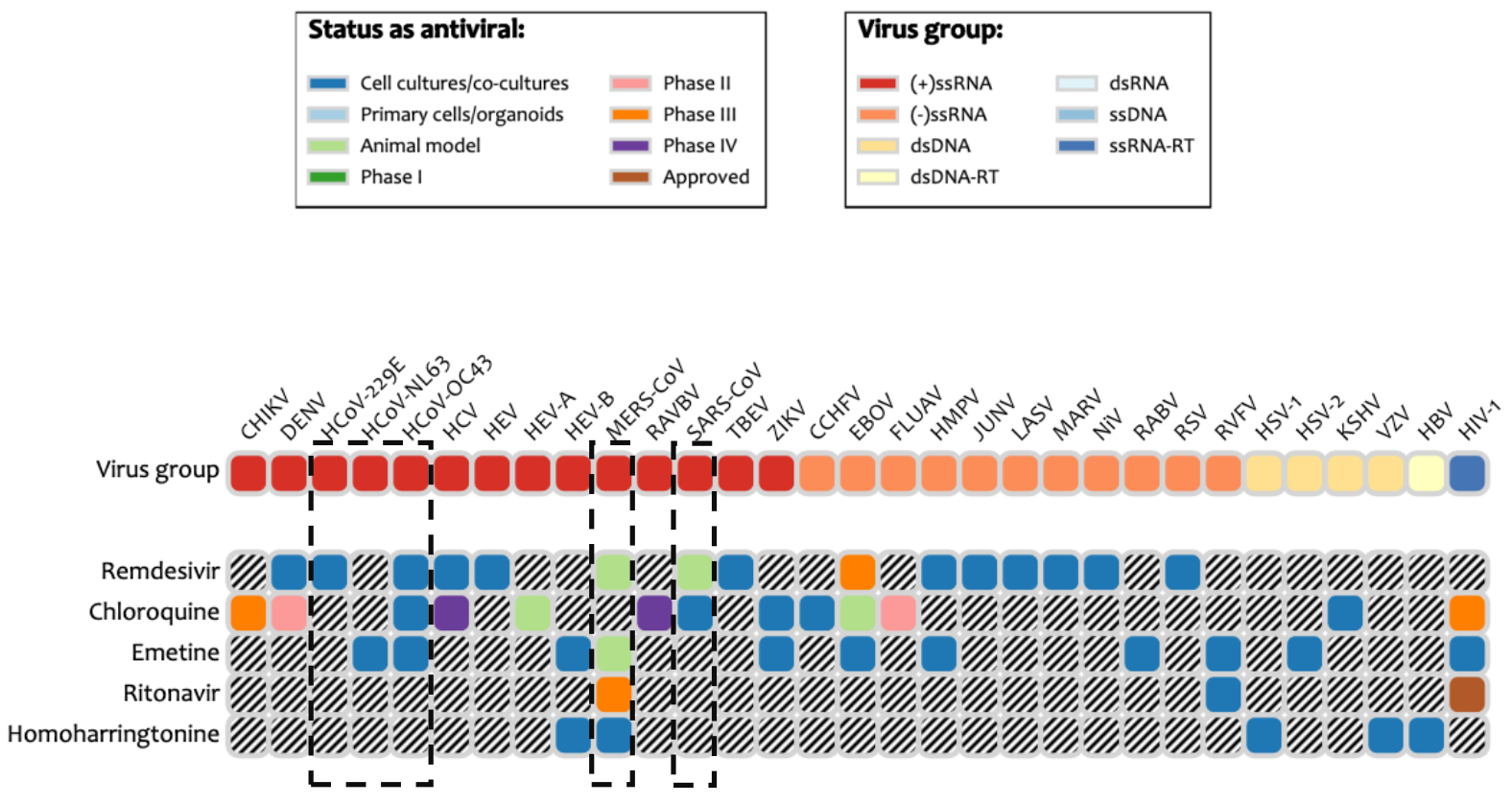

Fig. 3: Comparison of broad-spectrum antiviral activity of remdesivir, chloroquine and ritonavir which are currently undergoing clinical trials for the treatment of SARS-CoV-2 viral infection (COVID-19 disease) vs. emetine and homoharringtonine. Data was obtained from the broad-spectrum antiviral agents database, drugvirus.info. Highlighted by dashed lines are the activity of these drugs on different coronaviruses. Besides its antiviral activity, emetine is also an expectorant in cough syrups and has also shown promise for the treatment of pulmonary arterial hypertension (35). The drug may therefore have additional effects in patients with pulmonary complications such as COVID-19.

\section{Discussion}

It is important to point out a number of limitations of this study. First, the transcriptional response to exogenous RNA analyzed in this study is based on data from a single cell type (CD34+ HSPCs) while transcriptional responses to perturbations varies across cell types. Nevertheless, by comparing the transcriptional responses of HSPCs to those of 
cancer cell lines exposed to small molecules in the CMap database, it is evident some of the genes involved in innate antiviral defenses are highly coregulated across cell types (Fig. 2). Secondly, CD34+ HSPCs have a strong restriction to lentiviruses such as HIV-1 $(36,37)$, making it possible that the transcriptional responses to exogenous RNA in these cells could be stronger than that in other cells. While this may be the case, there appears to be a considerable extent of co-regulation of the genes involved across cell types. Finally, whether any of the identified drugs can be repurposed to novel viruses like SARSCoV-2 requires a deeper understanding of the drug bioavailability in relevant tissues, the relative tolerability given the disease severity, the cost and scalability in manufacturing which are not addressed in this study.

At least two mRNA-based vaccines are under development for COVID-19 by the companies Moderna Therapeutics and CureVac. These vaccines aim to elicit adaptive immunity which is commonly viewed as independent from the innate antiviral defenses considered in this study. Yet, it is becoming increasingly clear that long-lasting adaptive humoral and cellular immunity involves an interplay with innate immunity (38-40). While innate antiviral responses to foreign RNA can negatively impact expression of the encoded antigens in mRNA vaccines, some of the small molecules identified in this study may act as adjuvants to these vaccines. Since PRRs including those that detect foreign RNA are targets of many vaccine adjuvants $(38,39)$, these small molecules may increase the quantity and quality of immune responses to mRNA vaccines when co-delivered. Hence, we propose that the small molecules identified in this study should be explored 
both for inhibitory activity against SARS-CoV-2/ COVID-19 as well as for potential enhancement of immunological responses to mRNA vaccines including for COVID-19.

\section{Acknowledgements}

GHS is supported by the Center for Research Computing and the Eck Institute for Global Health at the University of Notre Dame.

\section{Author contributions}

GHS conceived and designed the study. Both GHS and AVN performed analysis. GHS wrote the manuscript. Both authors reviewed the manuscript.

Conflict of interest: The authors declare no competing financial interests in the work presented.

\section{Methods}

\section{Matching cas9 mRNA transcriptional responses to small molecules}

We obtained differentially expressed genes in CD34+ hematopoietic stem cells (HSPCs) exposed to cas 9 mRNA and sgRNA targeting the hemoglobin B locus (HBB) from Cromer et al (3). Briefly, the study by Cromer at al delivered cas9 mRNA containing 5methylcytidine and pseudouridine modifications which limit innate immune responses whereas the sgRNA had 2'-o-methyl-3'-phosphothioate modification to enhance tolerance and activity. The RNAs were delivered into cells using electroporation. Differential gene expression analysis was performed by computing log2 fold changes 
between cas 9 mRNA treated samples and those exposed to mock electroporation. We constructed a transcriptional signature for cas 9 mRNA response consisting of the top 150 upregulated genes and the 150 most downregulated genes with a cut-off value of MLogP (i.e. $-\log 10($ Pvalue) $>7$ ). This transcriptional signature was then used to query the CMap database which contains about 1.3 million transcriptional responses of human cell lines to chemical and genetic perturbation (2). We restricted the number of genes in the signature based on the recommended optimal signature size for querying the L1000 based CMap (2). To limit the analysis to well annotated genetic and small molecule perturbations in a core panel of cell lines, we queried the Touchstone dataset of CMap which contains about 238,125 expression signatures, approximately half of the total signatures in CMap. We performed two independent queries on CMap: i) the first query was to assess the similarity between the cas 9 mRNA signature and overexpression of different genes in human cells lines, ii) the second query was to assess the similarity between the cas9 mRNA signature and several small molecules. We performed queries using the Clue.io API data version 1.1.1.2 and software version 1.1.1.43.

\section{Filtering of predicted small molecules for downstream mRNA delivery effects}

To filter out small molecules whose transcriptional signatures may have matched that of cas9 mRNA delivery due to downstream effects (e.g. translation of the mRNA into cas9 protein that mediates double strand breaks), we also queried CMap using transcriptional signatures constructed from genes differentially expressed after delivery of cas 9 protein combined with sgRNA targeting HBB (ribonucleoprotein, RNP). The RNP signatures were constructed in a similar way as described above for cas9 mRNA and used to query CMap 
for small molecules with matching signatures. We then excluded small molecules whose signatures matched both cas9 mRNA and RNP signatures.

\section{Broad-spectrum antiviral analysis}

To determine broad-antiviral potential of the predicted small molecules on coronaviruses, we used public data obtained from high-throughput screens of 2000 compounds (29) and a smaller library of 290 approved drugs (30). We also obtained broad-spectrum activity data to other viruses from a recent study (28) and its associated database (drugvirus.info).

\section{References}

1. M. Schlee, G. Hartmann, Discriminating self from non-self in nucleic acid sensing. Nat. Rev. Immunol. (2016), , doi:10.1038/nri.2016.78.

2. A. Subramanian et al., A Next Generation Connectivity Map: L1000 Platform and the First 1,000,000 Profiles. Cell (2017), doi:10.1016/j.cell.2017.10.049.

3. M. K. Cromer et al., Global Transcriptional Response to CRISPR/Cas9-AAV6Based Genome Editing in CD34+ Hematopoietic Stem and Progenitor Cells. Mol. Ther. (2018), doi:10.1016/j.ymthe.2018.06.002.

4. B. Wienert, J. Shin, E. Zelin, K. Pestal, J. E. Corn, In vitro-transcribed guide RNAs trigger an innate immune response via the RIG-I pathway. PLoS Biol. (2018), doi:10.1371/journal.pbio.2005840.

5. T. Saito, D. M. Owen, F. Jiang, J. Marcotrigiano, M. Gale, Innate immunity induced by composition-dependent RIG-I recognition of hepatitis C virus RNA. Nature (2008), doi:10.1038/nature07106. 
6. S. M. Horner, M. Gale, Regulation of hepatic innate immunity by hepatitis C virus. Nat. Med. (2013), , doi:10.1038/nm.3253.

7. S. Kim et al., CRISPR RNAs trigger innate immune responses in human cells. Genome Res. (2018), doi:10.1101/gr.231936.117.

8. L. Kakkola et al., Anticancer compound ABT-263 accelerates apoptosis in virusinfected cells and imbalances cytokine production and lowers survival rates of infected mice. Cell Death Dis. (2013), doi:10.1038/cddis.2013.267.

9. V. Mgbemena et al., Transactivation of Inducible Nitric Oxide Synthase Gene by Kruppel-like Factor 6 Regulates Apoptosis during Influenza A Virus Infection. J. Immunol. (2012), doi:10.4049/jimmunol.1102742.

10. V. Mgbemena, J. Segovia, T. H. Chang, S. Bose, KLF6 and iNOS regulates apoptosis during respiratory syncytial virus infection. Cell. Immunol. (2013), doi:10.1016/j.cellimm.2013.06.002.

11. H. Poeck et al., Recognition of RNA virus by RIG-I results in activation of CARD9 and inflammasome signaling for interleukin 1B production. Nat. Immunol. (2010), doi:10.1038/ni.1824.

12. A. M. Kell, M. Gale, RIG-I in RNA virus recognition. Virology (2015), , doi:10.1016/j.virol.2015.02.017.

13. K. S. Bonham et al., A promiscuous lipid-binding protein diversifies the subcellular sites of toll-like receptor signal transduction. Cell (2014), doi:10.1016/j.cell.2014.01.019.

14. A. V Nordor, M. J. Aryee, G. H. Siwo, "Global Transcriptional Response to CRISPR/CAS9-AAV6 Based Genome Editing" Matches Transcriptional Response 
to Specific Small Molecule Perturbations. bioRxiv, 399311 (2018).

15. P. M. Yau, T. Godefroy-Colburn, C. H. Birge, T. V Ramabhadran, R. E. Thach, Specificity of interferon action in protein synthesis. J. Virol. (1978), doi:10.1128/jvi.27.3.648-658.1978.

16. T. V Ramabhadran, R. E. Thach, Specificity of protein synthesis inhibitors in the inhibition of encephalomyocarditis virus replication. J. Virol. (1980), doi:10.1128/jvi.34.1.293-296.1980.

17. H. J. Dong et al., The Natural Compound Homoharringtonine Presents Broad Antiviral Activity In Vitro and In Vivo. Viruses (2018), doi:10.3390/v10110601.

18. N. Khandelwal et al., Emetine inhibits replication of RNA and DNA viruses without generating drug-resistant virus variants. Antiviral Res. (2017), doi:10.1016/j.antiviral.2017.06.006.

19. S. Yang et al., Emetine inhibits Zika and Ebola virus infections through two molecular mechanisms: Inhibiting viral replication and decreasing viral entry. Cell Discov. (2018), doi:10.1038/s41421-018-0034-1.

20. B. Kroczynska, S. Mehrotra, A. D. Arslan, S. Kaur, L. C. Platanias, Regulation of interferon-dependent mRNA translation of target genes. J. Interf. Cytokine Res. (2014), , doi:10.1089/jir.2013.0148.

21. Y.-P. Liu et al., Endoplasmic Reticulum Stress Regulates the Innate Immunity Critical Transcription Factor IRF3. J. Immunol. (2012), doi:10.4049/jimmunol.1102737.

22. N. Kumar et al., Inhibitor of sarco/endoplasmic reticulum calcium-ATPase impairs multiple steps of paramyxovirus replication. Front. Microbiol. (2019), 
doi:10.3389/fmicb.2019.00209.

23. J. W. Perry et al., Antiviral activity of a small molecule deubiquitinase inhibitor occurs via induction of the unfolded protein response. PLoS Pathog. (2012), doi:10.1371/journal.ppat.1002783.

24. T. Carletti et al., Viral priming of cell intrinsic innate antiviral signaling by the unfolded protein response. Nat. Commun. (2019), doi:10.1038/s41467-01911663-2.

25. B. Gabrielsen et al., in Natural Products as Antiviral Agents (1992).

26. F. Wu et al., A new coronavirus associated with human respiratory disease in China. Nature (2020), doi:10.1038/s41586-020-2008-3.

27. N. Zhu et al., A Novel Coronavirus from Patients with Pneumonia in China, 2019. N. Engl. J. Med. (2020), doi:10.1056/NEJMoa2001017.

28. P. I. Andersen et al., Discovery and development of safe-in-man broad-spectrum antiviral agents. Int. J. Infect. Dis. (2020), doi:10.20944/preprints201910.0144.v5.

29. L. Shen et al., High-Throughput Screening and Identification of Potent BroadSpectrum Inhibitors of Coronaviruses. J. Virol. (2019), doi:10.1128/jvi.00023-19.

30. J. Dyall et al., Repurposing of clinically developed drugs for treatment of Middle East respiratory syndrome coronavirus infection. Antimicrob. Agents Chemother. (2014), doi:10.1128/AAC.03036-14.

31. E. de Wit et al., Prophylactic and therapeutic remdesivir (GS-5734) treatment in the rhesus macaque model of MERS-CoV infection. Proc. Natl. Acad. Sci. (2020), doi:10.1073/pnas.1922083117.

32. M. Wang et al., Remdesivir and chloroquine effectively inhibit the recently 
emerged novel coronavirus (2019-nCoV) in vitro. Cell Res. (2020), , doi:10.1038/s41422-020-0282-0.

33. M. L. Holshue et al., First Case of 2019 Novel Coronavirus in the United States. N. Engl. J. Med. (2020), doi:10.1056/nejmoa2001191.

34. P. Colson, J. M. Rolain, D. Raoult, Chloroquine for the 2019 novel coronavirus SARS-CoV-2. Int. J. Antimicrob. Agents (2020), , doi:10.1016/j.ijantimicag.2020.105923.

35. M. A. H. Siddique et al., Identification of Emetine as a Therapeutic Agent for Pulmonary Arterial Hypertension: Novel Effects of an Old Drug. Arterioscler. Thromb. Vasc. Biol. (2019), doi:10.1161/ATVBAHA.119.313309.

36. D. O. Griffin, S. P. Goff, Restriction of HIV-1-based lentiviral vectors in adult primary marrow-derived and peripheral mobilized human CD34+ hematopoietic stem and progenitor cells occurs prior to viral DNA integration. Retrovirology (2016), doi:10.1186/s12977-016-0246-0.

37. J. Zhang, D. T. Scadden, C. S. Crumpacker, Primitive hematopoietic cells resist HIV-1 infection via p21 Waf1/Cip1/Sdi1. J. Clin. Invest. (2007), doi:10.1172/JCl28971.

38. R. L. Coffman, A. Sher, R. A. Seder, Vaccine adjuvants: Putting innate immunity to work. Immunity (2010), , doi:10.1016/j.immuni.2010.10.002.

39. E. Nanishi, D. J. Dowling, O. Levy, Toward precision adjuvants: Optimizing science and safety. Curr. Opin. Pediatr. (2020), , doi:10.1097/MOP.0000000000000868.

40. M. G. Netea, A. Schlitzer, K. Placek, L. A. B. Joosten, J. L. Schultze, Innate and 
Adaptive Immune Memory: an Evolutionary Continuum in the Host's Response to Pathogens. Cell Host Microbe (2019), , doi:10.1016/j.chom.2018.12.006. 